\title{
LA OBJECIÓN DE CONCIENCIA DE LOS PROFESIONALES Y SU RELACIÓN CON LA ATENCIÓN EN SALUD SEXUAL Y REPRO- DUCTIVA DE ADOLESCENTES EN SANTIAGO DE CHILE
}

\author{
Adela Montero Vega ${ }^{1}$
}

Resumen: En la práctica clínica, el conflicto por la objeción de conciencia emerge al confrontarse el derecho del profesional objetor a la libertad de conciencia y el derecho de las personas a la atención en salud, oportuna, de calidad y sin discriminación. Frente a una objeción resulta imprescindible considerar la existencia de requisitos y límites, descartando su utilización para eludir la responsabilidad profesional o como forma encubierta de prácticas discriminatorias.

Mediante un estudio exploratorio, cualitativo, se analizaron los principales significados y aspectos bioéticos relacionados con la objeción de conciencia profesional en la atención de salud sexual y reproductiva de adolescentes de la Región Metropolitana, en Santiago de Chile.

Los resultados evidencian que, en la medida que se establezcan las bases éticas y legales para resguardar los derechos fundamentales, basados en el respeto por la dignidad de todos los involucrados, podremos lograr un real entendimiento entre las partes que a menudo entran en conflicto respecto de esta materia.

Palabras clave: objeción de conciencia, bioética, adolescencia, salud sexual y reproductiva

\section{Conscientious objection of professionals and its relation to sexual and reproductive health care of adolescents in Santiago of Chile}

\begin{abstract}
In clinical practice, the conflict due to conscientious objection arises when confronting the right of professional objection to free conscience and the right of persons to health care on time, with quality and without discrimination. When facing an objection is indispensable to consider the existence of requirements and limits, ruling out its use to evade professional responsibility or as a way to hide discriminatory practices.

Using an exploratory, qualitative study, the main meanings and bioethical issues related to professional conscientious objection were analyzed in sexual and reproductive health care of adolescents of Metropolitan Region in Santiago of Chile.

The results show that, in the extend that ethical and legal basis safeguard fundamental rights, based in the respect for the dignity of all involved, we may achieve a real understanding between the stakeholders that often enter into conflict with respect to this issue.
\end{abstract}

Key words: Conscientious objection, bioethics, adolescence, reproductive and sexual health care

A objeçáo de consciência dos profissionais e sua relaçáo com a atençáo em saúde sexual e reprodutiva de adolescentes em Santiago do Chile

Resumo: Na prática clínica, o conflito pela objeção de consciência emerge ao confrontar-se o direito do profissional objetor à liberdade de consciência e o direito das pessoas à atenção em saúde oportuna, de qualidade e sem discriminação. Frente a uma objeção resulta imprescindível considerar a existência de requisitos e limites, descartando a sua utilizaçáo para eludir a responsabilidade profissional ou como forma encoberta de práticas discriminatórias.

Mediante um estudo exploratório, qualitativo, se analisaram os principais significados e aspectos bioéticos relacionados com a objeção de consciência profissional na atenção à saúde sexual e reprodutiva de adolescentes da Região Metropolitana, em Santiago do Chile.

Os resultados evidenciam que, na medida que se estabelecem as bases éticas e legais para resguardar os direitos fundamentais, baseados no respeito pela dignidade de todos os envolvidos, poderemos lograr um real entendimento entre as partes que com frequência entram em conflito a respeito desta matéria.

Palavras-chave: objeção de consciência, bioética, adolescência, saúde sexual e reprodutiva

\footnotetext{
${ }^{1}$ Centro de Medicina Reproductiva y Desarrollo Integral de la Adolescencia, CEMERA, Facultad de Medicina, Universidad de Chile, Chile Correspondencia: amontero@med.uchile.cl
} 


\section{Introducción ${ }^{2}$}

Desde la antigüedad, la objeción de conciencia (OC) ha sido un tema complejo, presente en la discusión ética, al aludir a lo más íntimo del ser humano, como es su dignidad, basada en los dictámenes de su conciencia, llegándose a plantear que debería ser considerada un derecho fundamental de las personas, de rango constitucional(1). Aun cuando en la historia existen variados ejemplos(2), solo a comienzos del siglo XX, principalmente en Europa, surge como entidad reconocible convencionalmente, como oposición para desarrollar obligaciones de naturaleza militar(3) y participar directamente en conflictos bélicos(4), manifestándose posteriormente las primeras evidencias explícitas en el campo de la salud(4).

El concepto relativo a la $\mathrm{OC}$ es considerado desde diversas posturas, determinando cierto grado de imprecisión y de confusión. Algunos autores la consideran una manifestación de incumplimiento de una obligación de naturaleza legal, cuyo acatamiento o realización produciría en la persona una grave lesión de su conciencia, con base en motivaciones de índole ética, moral, religiosa, axiológica o de justicia del individuo objetor(5,6). También ha sido considerada como un derecho subjetivo, de excepción, ante una obligación o normativa legal, con incumplimiento de esta por razones religiosas o filosóficas, derivada de las relaciones laborales o funcionarias de un individuo(7). Si consideramos la conciencia, perteneciente solo a la esfera individual, inherente al ser humano, es posible entender la $\mathrm{OC}$ como la negación vinculada al contexto privado o particular, planteándose como una obligación personal, no pudiendo ser de índole colectiva ni institucional(7-9). Concomitantemente, se la señala como aquella manifestación individual, separada de la desobediencia civil, por parte del personal de salud, del paciente e incluso de los familiares, reconocible como un derecho, siempre que su aplicación no afecte o lesione a terceros $(4)$.

\footnotetext{
${ }^{2}$ Trabajo correspondiente a Tesis para obtener el Grado de Magíster en Bioética de la Universidad de Chile: "Objeción de conciencia sanitaria en profesionales relacionados con la atención de salud sexual y reproductiva en adolescentes de la Región Metropolitana, Santiago de Chile".
}

Pese a existir algunos acuerdos en el ámbito internacional sobre la definición, alcances y restricciones a la libertad de conciencia en los profesionales sanitarios, las observaciones empíricas no sistematizadas indican que, en la práctica, estos profesionales orientan sus acciones sobre la base de una diversidad de significados respecto a la OC. Como ello tendría innegables repercusiones éticas en la relación con los pacientes y con la sociedad, resulta interesante y necesario investigar sobre esta realidad en nuestro país, en un camino para llegar a establecer posibles acuerdos para regular estas prácticas, considerando la experiencia y la percepción de los profesionales directamente implicados. Por ello, este trabajo pretende describir y analizar las principales nociones, argumentaciones y experiencias, así como las posibles implicancias bioéticas que pudieran presentarse entre los profesionales de la salud respecto a la OC y su relación con la atención clínica especializada en salud sexual y reproductiva de adolescentes (SSRA) de la Región Metropolitana, Santiago de Chile.

\section{Métodos}

Se realizó un estudio exploratorio de diseño cualitativo. Desde una perspectiva fenomenológica, la investigación cualitativa fue estimada la más idónea, porque permite al investigador que está inmerso dentro de un contexto llegar a comprender con mayor profundidad un fenómeno parcial o insuficientemente investigado como es la materia que nos ocupa (10). De este modo, el conocimiento surge a partir de la propia percepción de las personas involucradas en la temática que se quiere conocer, permitiendo un acercamiento más dinámico a la construcción del fenómeno a investigar, mediante la obtención y análisis de contenidos, opiniones, acciones y/o significados personales asignados a la experiencia personal(10-14).

Se elaboró un registro de los profesionales que trabajan en centros o unidades de salud públicos y privados especializados en la atención en SSRA de la Región Metropolitana, procediendo a seleccionar una muestra teórica o propositiva, incluyendo como unidades de muestreo a aquellos que por sus características o perfiles fueron considerados informantes clave, es decir, quienes mejor pudieran contribuir a la explicación del fenómeno a estudiar. Se consideraron aspectos como: edad, 
género, profesión y años de experiencia de trabajo con adolescentes. Según criterio de saturación, se determinó el tamaño o suficiencia del grupo de estudio, concepto que alude a la cantidad y calidad de la información necesaria para considerar el tamaño muestral. La muestra se consideró suficiente cuando al conjunto de la información obtenida de manera acumulativa, no fue posible agregar un mayor conocimiento, es decir, cuando la información conseguida de las siguientes unidades de muestreo se volvió repetitiva, redundante, sin aspectos nuevos, constatándose agotamiento del contexto y/o de los contenidos(12,15). Es importante explicitar que el poder de la muestra se fundamentó en la calidad, riqueza y profundidad de la información aportada $(12,15)$. Como técnica de recolección de la información se recopilaron los antecedentes sociodemográficos de los entrevistados.

A continuación se realizó entrevista en profundidad semiestructurada, individual y confidencial, con preguntas abiertas, utilizando una guía o pauta previamente diseñada, para no olvidar aspectos importantes relacionados con el estudio, conservando la flexibilidad necesaria en el trabajo de campo $(10,12,16)$. Al momento de la entrevista, se aclararon las dudas y se revisó y firmó el consentimiento informado. Los contenidos fueron registrados con grabadora de voz, debidamente autorizada por los entrevistados y por notas de campo. Se resguardó la confidencialidad de los datos, con acceso restringido a los formularios, grabaciones, transcripciones y bases de datos. La información transcrita se procesó con ayuda del programa AQUAD 5. Siguiendo el modelo de la Teoría Fundamentada de Glasser y Strauss $(11,13)$, se efectúo análisis de contenido de tipo semántico, poniendo énfasis en el contenido del texto, evaluando el sentido de las palabras y analizando los temas y categorías propuestas para el análisis $(14,17)$. Se contó con la aprobación del Comité de Ética de Investigación en Seres Humanos de la Facultad de Medicina de la Universidad de Chile.

\section{Resultados}

Entre el 28 de diciembre de 2010 y el 5 de julio de 2011 se completó un total de 16 entrevistas, alcanzando saturación de la información. Cada entrevista duró en promedio 53,2 minutos (3177 minutos).

En el trabajo de campo se preguntó cómo sería la percepción, conocimiento, creencias, argumentos, experiencias y aspectos bioéticos respecto a la OC de los profesionales relacionados con la atención en SSRA.

De manera didáctica, la información será presentada aquí de acuerdo a cómo fue procesada y ordenada según el siguiente orden analítico: características del grupo de estudio, procesamiento de la información y análisis de resultados.

1. Características del grupo de estudio: la totalidad de las personas entrevistadas fueron de sexo femenino. Hubo 12 médicos (cinco gineco obstetras especialistas en ginecología adolescente; seis pediatras especialistas en adolescencia, un psiquiatra infanto juvenil), dos matronas, una psicóloga infanto juvenil y una asistente social. La edad promedio fue de 43,8 años (30-63 años). El promedio de años de experiencia profesional fue 19,8 años (6-39 ańos). La experiencia promedio de trabajo con adolescentes fue 15,6 años (5-30 años). Del total de profesionales, ocho se desempeñan en el sistema público y privado, cinco en el sistema privado y tres en el sistema público. Once personas tenían experiencia previa o actual en cargos directivos, ocho de ellas mayor a cinco años. Respecto de la religión, la mayoría se considera católica y dos personas manifestaron no profesar ninguna creencia.

2. Procesamiento de la información: con el programa AQUAD 5 se realizó codificación lineal, asignándole un carácter nominal a cada línea de la entrevista transcrita. Posteriormente, se realizó codificación analítica, mediante lectura crítica de cada texto codificado linealmente de cada una de las entrevistas, procediendo a seleccionar los párrafos que permitieran acceder al universo de los significados de estos 16 actores claves entrevistados. Los principales códigos construidos a partir de la lectura de estas transcripciones fueron: noción, argumentos, importancia, OC del paciente, OC del profesional, ejemplos OC, requisitos, sistema de atención público/privado, situación laboral, experiencia, deber profesional, características equipo, reacción equipos, relación profe- 
sional/institución, rol institucional, rol profesional, aspectos bioéticos, vulnerabilidad pacientes, conflictos bioéticos, consecuencias, género y OC, límites OC, relación profesional/paciente, relación profesional/equipo, derechos del paciente, derechos del profesional, respaldo jurídico, validez, excepciones, democracia, respuesta sociedad, génesis $\mathrm{OC}$, posibles soluciones, autocrítica, ética de mínimos, ética de máximos.

Esta codificación permitió cumplir con el primer paso del análisis, consistente en reducción de la información, para proceder a la elaboración de las categorías temáticas para la organización de los datos. Es importante señalar que la categorización alude a una clasificación conceptual, abstracta, de unidades que son cubiertas o sustentadas por un significado común, mientras que la codificación permite identificar qué partes del discurso serán consideradas en una categoría(14).

3. Análisis de resultados: las principales categorías identificadas fueron: "Noción"; "Argumentos"; "Experiencia"; "Dimensión bioética" y "Cursos de acción como herramientas para superar las diferencias asociadas a la OC”.

Durante la elaboración y construcción de categorías, cada una fue definida operacionalmente, procediendo a distinguir las principales subcategorías, definidas como las propiedades que sustentan la categoría.

A continuación se hará referencia al análisis de contenido semántico previamente descrito, en el cual el sistema de categorías y subcategorías constituyen un mapa de significados que representan la estructura de contenido de las entrevistas realizadas. El análisis reflexivo basado en este sistema permitió generar la Teoría Fundamentada. En una primera etapa, el análisis fue desarrollado sobre la base de las principales categorías con sus correspondientes subcategorías, comenzando con el análisis de los principales aspectos contenidos en cada una de ellas.

NOCIÓN: se definió como el conocimiento o idea elemental que se tiene sobre alguna materia o condición(18).
Para la mayoría de los profesionales, comprendería múltiples y variados significados. Se relacionaría con la negación, impedimento, limitación o interferencia a una determinada acción, atención, actividad o procedimiento en salud, por contraposición con las creencias o valores del profesional: "cuando por una... cuando por, necesito negarme a dar una atención en salud debido a que se contrapone absoluta y completamente con mis creencias personales" (E10)3; "sería algo como que te impide, una creencia, quizás basada en ciertos principios religiosos o valóricos, que te impide realizar una actividad o un procedimiento o entregar una atención médica a la persona que me está consultando por ese motivo" (E15).

Otros la consideran una forma directa de incumplimiento a una normativa o medida legal, por contraposición con los valores del profesional responsable de entregar la prestación: "el médico puede ehh... no cumplir con una... medida que está estipulada en la ley, por razones que aluden a sus valores, o en el fondo a su conciencia" (E12); "lo que yo entiendo como $O C$, de poder eh, objetar la aplicación de determinadas normativas o leyes, normativas en términos más generales, amparados bajo una, un fundamento que estaba ligado a lo valórico, o sea a su conciencia" (E8).

También es concebida como una incompatibilidad, una contraposición que surge entre la obligación que emana de la profesión y los valores personales: "si la llevo a cabo, la atención por la que se objeta en conciencia, realmente estoy cometiendo para mis creencias personales, un daño, a mi mismo o a mi alma, o un pecado tan importante que en realidad es algo que no puedo, no puedo realizar, es una, es una palabra, una contraposición, pero tan importante, entre mi deber ser o mi obligación como médico, con mis valores personales, que es tan incompatible, que en realidad no lo puedo... no lo puedo llevar a cabo" (E10).

Estaría vinculada con la capacidad o competencia del objetor, para decidir libremente sobre actitudes, responsabilidades o indicaciones relacionadas con un acto médico: "para mi cumple los mismos o sea tiene la misma definición de cualquier OC en el ámbito médico, quizás en otros ámbitos es diferente, pero en el ámbito médico la OC es... la capacidad

${ }^{3}\left(\mathrm{E}=\right.$ entrevista; $10=\mathrm{n}^{\circ}$ asignado a la entrevista $)$ 
de rechazar o no cumplir, no es rechazar, es la capacidad de no cumplir..." (E12).

Se identifican diversos agentes involucrados, surgiendo diferencias al momento de considerar quiénes son los principales actores. Para la mayoría, incluiría solo a profesionales directamente relacionados con una acción específica: "creo que es la persona que directamente tiene que hacer una acción, asi como concreta, que es la que sería, la que estaría con más responsabilidad, el resto debería cumplir lo que tiene que hacer no más, a mí me parece" (E4).

Otros, según la complejidad de la situación particular, identificarían una mayor cantidad de involucrados, por ejemplo, en el caso del aborto, la OC pudiera ser planteada por la totalidad de las personas que tomen conocimiento: "lo que pasa es que si tú estás trasladando para que haga su aborto, eso si, se puede hacer una OC" (E11)... "si pueden objetar en conciencia, porque todo lo que hagas para facilitar el procedimiento por el cual tú no estás de acuerdo, ya sea la matrona que ingresa, la pabellonera que la lleva, la anestesista que le pone la anestesia... el chofer, sí, todos" (E11).

Para algunos, comprendería la totalidad del proceso en una determinada prestación, desde la entrega de la información a la ejecución del procedimiento: "puede ser a todo, puede ser una indicación, o sea no necesariamente a un procedimiento, sino que una indicación, un acto terapéutico que incluye la indicación hasta el procedimiento" (E5); "desde la entrega de información, claro, estaría involucrada entonces la entrega de información, el mismo hecho de hacer la receta, claro, tengo entendido que sí, todo" (E5).

La noción abarcaría solo el ámbito personal, por ende no incluiría a las instituciones: "la OC debiese acotarse al ámbito de las decisiones individuales, yo creo que en ese ámbito es perfectamente respetable, pero que eso determine la conducta que va a tener toda una institución que se inserta en un pais, donde se necesitan implementar determinadas medidas de salud pública por el bienestar de la población, y que eso esté determinado asi a nivel institucional, me parece que es inadecuado..." (E8).
Globalmente, se la reconoce como un derecho, condicionado al cumplimiento de ciertos requisitos como: profesional directamente involucrado; conducta irreprochable; actuar moralmente congruente; necesidad de explicitar la OC a los superiores y al equipo; derivación; entrega de la información; definición precisa; disponibilidad de recursos: "el derecho y la posibilidad que un profesional puede, tiene, de negarse a participar en alguna intervención por motivos éticos, morales, personales" (E5).

Existe una percepción negativa, al estimarla como una maniobra encubierta para eludir la responsabilidad profesional: "creo que mucha gente que trabaja con adolescentes lo utiliza sin sentirla realmente, sino que como una excusa como pa'sacarse el problema de encima"...(E6); o al considerarla un error, una equivocación en el desempeño profesional: "...porque probablemente la situación más, la unica situación que me ha llevado a eso, precisamente a eso, es decir, yo no puedo con errores, es la situación del aborto, pero no justifico mi accionar porque después logré comprender que eso no tiene que ver que ese paciente tiene igual el derecho a la información" (E6). También surge una descalificación explícita al vincularla con fundamentaciones religiosas: "hay mucha gente que cree que objetar en conciencia es ehhh es algo... a ver, hay gente que lo ve súper negativo ya, porque que es como de flojos o que es como de gente cerrada, ultra católica, porque casi siempre en ese contexto se ponen las cosas, y que no está capacitada para atender adolescentes, porque además va a comprometer la atención del adolescente..." (E11).

ARGUMENTOS: operacionalmente, fue definida como el razonamiento empleado para demostrar la explicación de un concepto o materia determinada.

Para la mayoría de los participantes, los principales argumentos estarían directamente vinculados con un fundamento religioso, católico, resultando difícil la identificación de otros: "como que siempre OC yo la he visto casi como sinónimo, pensando que en el fondo los únicos que pueden tener objeciones de conciencia son aquellos cuyas creencias religiosas ehhh, de alguna manera están contrapuestas o contraviniendo todo lo que es lo, la tendencia actual en la atención en SSR y en anticoncepción moderna, 
etc., entonces pa' mí casi siempre, así, pensar en objeción de conciencia ha sido aquellos que profesan la ehhh, siendo cristianos, sean católicos, católicos apostólicos y romanos, porque para mí personalmente prácticamente no hay ninguna otra religión que tenga OC" (E10); "la verdad es que no tengo claro otro, estoy pensando si podria haber no sé, algún fundamento no sé político a lo mejor, no sé, lo tengo poco claro en ese aspecto, yo siento que en general tiene que ver más con esta cosa que es propia más de la cosa religiosa, de verdad, que es algo que nos marca a las personas muy profundamente" (E4).

Es importante consignar que aquellos profesionales que de manera explícita se consideraban como objetores, habitualmente poseían un argumento religioso que fundamentaba su objeción: "para mi que soy católica, igual hay veces en que hay ciertas ehhh... no sé si prácticas, pero ciertas cosas que van en contra de lo que yo creo..." (E11); "...lo que como médico, uno como profesional le puede ofrecer, sin caer en cosas que a lo mejor tienen que ver con lo personal de uno, con la religión que uno tiene, cosa que yo creo que es más como lo que me pasó, me enredaba, me complicaba mi religión, por ejemplo en relación a sexualidad y tener que indicar algún método anticonceptivo" (E9).

Por el contrario, la mayoría, al no existir esta premisa, no se considera objetor. Sin embargo, se logra identificar implícitamente argumentos basados en los aspectos éticos de la relación clínica: "por ejemplo, cuando se planteó el tema si teníamos que denunciar o no a las adolescentes menores de 14 años, porque habia que denunciarlo, porque la ley decía que habia que denunciarlo, porque están teniendo actividad sexual o nosotros sospechamos que tenian actividad sexual, y de acuerdo a ciertos decretos del servicio de salud, habia un decreto que me acuerdo perfectamente que decía que habia que denunciarlo, porque esto era un delito, pero ahi yo creo que es como la primera vez que empezamos a plantearlo como equipo y empezamos a discutir este tema y a tomar decisiones respecto a eso, tomar decisiones, digamos, qué era lo más beneficioso en este caso para la adolescente, si habia que denunciarla, entonces a que la exponíamos, la dejábamos fuera de la atención, la dejamos mucho en peores condiciones o veíamos como actuábamos" (E4).
EXPERIENCIA: se definió como vivencias que, a través de la práctica, permiten obtener el conocimiento sobre un determinado hecho, circunstancia o acontecimiento. Involucra diferentes situaciones o circunstancias en concordancia con: vivencias de los entrevistados, experiencia al interior de los equipos y experiencias relacionadas con el aspecto laboral.

Las principales situaciones se describieron con la anticoncepción en general, particularmente en menores de edad y con anticoncepción de emergencia: "por ejemplo, en el tema de la salud sexual, la anticoncepción, dar anticoncepción a las adolescentes" (E2); “en qué situaciones se ha planteado la OC?, ahh perfecto, bueno yo creo que son evidentes. La primera y la más frecuente se plantea cuando hay profesionales que consideran que no deben indicarle métodos anticonceptivos a una adolescente por su temprana edad" (E16); "con la anticoncepción de emergencia, que es uno de los casos en que se plantea" (E5).

Otros ejemplos se presentaban asociados al aborto, la confidencialidad en la atención clínica, la esterilización, la adopción de hijos de madres adolescentes y la orientación sexual homosexual: "anticoncepción, aborto, el que haya actividad sexual sin que sepan los padres, o sea, ese es un punto importante, está la confidencialidad entre los 14 y los 18, yo creo que ese es un tema, obviamente infecciones de transmisión sexual, sobretodo, o sea sin ehh, cuando no, no saben los padres y no quieren que sepan los padres, o sea sospecho y diagnostico una ITS ${ }^{4}$ en un adolescente que no quiere que se le informe a los padres, o sea son situaciones que pueden ser complejas y que pudieran crear, ser motivo de OC en algunas personas" (E10); "la anticoncepción, la esterilización... los abortos obviamente..." (E3); "en otros temas que abarcan la SSR no solo la actividad sexual y anticoncepción, también he visto de alguna manera gente muy activa en intentar generar un cambio de opinión en una adolescente, por ejemplo, que ha decidido entregar su hijo en adopción..." (E16); "también me he podido dar cuenta que hay gente que la siente incluso frente a pacientes, por ejemplo, que tienen una orientación sexual homosexual y que eso no va frente a su parada de vida..." (E6).

\footnotetext{
${ }^{4}$ Infección de transmisión sexual.
} 
Las experiencias al interior de los equipos de trabajo se asociaban a vivencias positivas y negativas. Las positivas derivaban del respeto mutuo entre el objetor y no objetores; en la necesidad de reflexionar, de dialogar y tomar decisiones en conjunto sobre los problemas; con equipos flexibles, con readecuación de funciones: "en el equipo, qué es lo que entra en juego ahi, qué es lo que... la capacidad de dialogar, la capacidad de conversar, la capacidad de entender, la capacidad de ponerse en el lugar del otro..." (E7); "creo que la honestidad y la madurez para asumirlo como equipo, es lo que yo respeto, que tenian una solución; en cambio hay otros equipos donde nadie sabe qué piensa el otro, o si ocurren estos casos sin querer se perjudica al paciente que está buscando una ayuda" (E9).

Las experiencias negativas fueron reportadas como situaciones de rechazo explícito, descalificatorias, con condicionamiento del contrato y permanencia laboral del objetor, secundario a la percepción de incompatibilidad con el pensamiento de los pares o con el perfil de la institución: "hay una persona del equipo que manifiesta que no puede indicar anticoncepción a adolescentes porque está en un culto religioso determinado y ese culto religioso no permite la anticoncepción en ninguna persona, tampoco adolescente ehhh, y desde ahi el tema se conversa con otras profesionales, hablando de si es válido o no que la persona siga trabajando en (...), porque en el fondo es una, esta religión a la que ella adscribe está en contra de varios aspectos de la sexualidad adolescente y no solo de la anticoncepción, entonces como que sería un poco contradictorio, no sería consecuente que ella estuviera en este lugar de trabajo" (E2); "esto lo planteé directamente en una reunión, hubo reacciones bastante duras, de hecho uno de nuestros colegas se paró y dijo que: 'la doctora no puede enseñar nunca más anticoncepción', se paró y se fue, otra persona... encontró que entonces yo no podria seguir trabajando con adolescentes, o sea... hubieron reacciones bien... bien duras en ese minuto, y además ocurrió que una persona dentro de esa misma reunión se dio cuenta de lo mismo que estaba pasando, e hizo el comentario de que en realidad 'oye calmemos esta cosa', no... y no fue tampoco, ni ese comentario, ni ese apoyo fue escuchado, o sea la gente siguió un poco, entre comillas, con una agresividad pasiva, a lo mejor no hay combo, no hay insultos, pero es agresión, es primero descalificación, o sea in- mediatamente fui descalificada como posible educadora, por decirlo de alguna forma, por una creencia personal..."(E3).

DIMENSIÓN BIOÉTICA: definida como la relación entre la OC y los principios bioéticos involucrados en la atención en SSRA.

La mayoría no logró definir ni dimensionar directamente los efectos o consecuencias de la OC como conflictos o repercusiones de carácter bioético. Los hallazgos surgieron al analizar el relato de situaciones conflictivas, advirtiéndose presencia de conflicto de valores en el ámbito personal, al interior de los equipos y en la relación con los pacientes o con las instituciones.

Formando parte de esta dimensión, se identificaron situaciones donde la OC profesional produjo un efecto contrario al esperado por el paciente, derivando incluso en un daño directo: al negar una prestación; frente a imposición de valores a adolescentes vulnerables; situaciones de maltrato directo mediante acciones ofensivas y circunstancias en las que la OC de una institución limitó el acceso y libertad de elección en la atención.

Aparece la necesidad de reconocer la existencia de límites que, por su importancia y significación, imponen la necesidad de excepciones a la OC, otorgándole un mayor nivel de exigencia que trascienda los requisitos previamente enunciados, como: respeto por los derechos del paciente; situaciones de riesgo vital; imposibilidad de derivación; entrega incompleta, incorrecta o no oportuna entrega de información.

Igualmente, en el discurso de la mayoría de los participantes emerge de manera rotunda la percepción de consecuencias que afectarían al profesional en el plano emocional, en su conciencia moral y en el medio laboral, asociadas nuevamente al condicionamiento contractual y permanencia del objetor: "entonces, te fijas que la imposición desde... puede dañar, yo creo que puede dañar al profesional en la parte personal, humana y formativa".. (E9; "yo creo que si uno lo explica... yo no soy una máquina que entrega una receta, yo soy una persona, que tengo sentimientos, que tengo valores, que tengo todo, entonces si uno es capaz de decirle por qué uno hace o no hace las cosas, deberian 
tomarlo bien" (E11); "ahi yo creo que si a mi me hubiese tocado que me hubiesen preguntado "tú das anticoncepción de emergencia", si yo le hubiese dicho en ese minuto que no, me habría pasado lo mismo, me habrian echado a mi..." (E11).

Asimismo, se identifican consecuencias que afectan al paciente, derivadas de la no entrega de una prestación, particularmente asociadas al género y nivel socioeconómico: "lo que he podido observar a veces, en otras personas cercanas a mí, que están muy sesgadas en eso y que tienen una posición muy drástica respecto a eso, y eso hace, no es cierto, que sesgue toda la intervención o atención que estoy haciendo con la persona, incluso de negar beneficio a la persona, de no darle la información correcta respecto a un tema, por ejemplo, ya y eso..., o sea lo he visto muy de cerca" (E4); "para qué hablar del problema de inequidad, de la chiquilla de escasos recursos y la que tiene la plata pa'ir a comprarla a la farmacia..." (E6); "es un tema que afecta a las mujeres, o sea a las poblaciones más vulnerables, a las mujeres y a las jóvenes, en general..." (E16).

Es importante destacar que no se logró identificar asociación entre las categorías y las características sociodemográficas de los informantes claves. Más que a determinantes sociales, las diferencias responderían a condicionantes personales, como por ejemplo respecto del propio reconocimiento como objetores. Los profesionales que se reconocían como tales, principalmente aludían un componente religioso en su fundamentación, asociado con la formación y práctica comprometida de un determinado credo o religión. Al contrario, aquellos que no se reconocían como objetores basados en un argumento religioso, tendían a ser más categóricos, surgiendo el deber y responsabilidad profesional como una máxima que era necesario cumplir, imponiéndose como una obligación, superior a la OC.

CURSOS DE ACCIÓN COMO HERRAMIENTAS PARA SUPERAR DIFERENCIAS ASOCIADAS A LA OC: fue definida como las estrategias para lograr posibles soluciones a través del entendimiento entre las partes involucradas, para superar las diferencias o conflictos que pudieran resultar de una OC. Surge de las siguientes subcategorías: autocrítica del profesional; marco regulador para superación de conflictos; cons- trucción democrática; respeto por la diversidad de posturas morales; necesidad de diálogo y formación en bioética.

La autocrítica apuntaba al juicio reflexivo sobre los valores en el desempeño o comportamiento del profesional durante la atención clínica, en la que el profesional constantemente pudiera reflexionar sobre su accionar, como una forma de evaluación interna.

La necesidad de un marco regulador para superar los conflictos, hace referencia a acuerdos sociales que estipulan mínimos de justicia para la convivencia entre extraños morales, susceptibles de expresión legal (ética de mínimos). Existe la impresión que estos mínimos de justicia, basados en leyes o normativas, serían de importancia al contribuir como una ayuda, como un respaldo del actuar profesional, que favorecería positivamente la relación clínica mediante normas claras en las que se explicitarían los principales aspectos relacionados con la OC. Pero también se reflexiona en cómo se evaluaría el incumplimiento del profesional objetor de las normativas previamente establecidas.

La necesidad de que, como país, podamos avanzar hacia una real construcción democrática, como característica de una sociedad pluralista, facilitaría la solución de los conflictos derivados de la OC.

El respeto por la diversidad de posturas morales aparece formando parte de una ética de máximos, al abogar por el respeto de la OC y con ello a la persona que realiza la objeción, como una máxima moral que alude a la libertad de conciencia y que se relacionaría con la apertura y flexibilidad para empatizar y aceptar las diferencias entre objetores y no objetores.

La necesidad de diálogo y deliberación al interior de los equipos consistiría en el debate reflexivo, mediante un proceso que permita vislumbrar y comprender una determinada situación desde varios puntos de vista, facilitando la toma de decisiones de manera más asertiva.

La formación en bioética emerge de manera explícita como herramienta para contrarrestar de manera objetiva los conflictos asociados a este 
problema. La insuficiente formación en la preparación profesional de pregrado y posgrado debiera revertirse a través de un perfeccionamiento eficaz, como una manera de facilitar el respeto por la diversidad y entendimiento de las diferencias, principalmente valóricas, para un adecuado resguardo de los aspectos éticos en la atención clínica de adolescentes en SSR.

\section{Discusión}

Los resultados ponen en evidencia la complejidad de la OC y sus diferentes matices, generando a menudo situaciones conflictivas, difíciles de conciliar y resolver. Nuestra elaboración teórica encuentra su marco de referencia en el encuentro y respeto por el otro, aun con lo perturbador que pudiera llegar a ser(11), particularmente cuando atañe a nuestros valores y/o creencias.

Si bien la OC debe estar enmarcada en el respeto por la dignidad humana de los involucrados, la evidencia presentada demuestra que no podría ser absoluta, debiendo plantearse como una medida excepcional, con la necesaria evaluación de requisitos y límites para su ejecución, respetando, como un bien superior, el derecho a la vida y salud de la población que pudiera verse afectada. Para ello es esencial establecer que no corresponda a una banalización de la libertad de conciencia, simplificando la toma de decisiones sin la adecuada reflexión sobre los argumentos o fundamentos que motivan la objeción(19).

Por ello, frente a una determinada OC, debiera evaluarse en primer lugar si es genuina, que no esté motivada por intereses particulares, ajenos a la dimensión moral. La tarea de verificar tal validez podría ser responsabilidad de los comités de ética clínicos, como mecanismo regulatorio de carácter imparcial(19).

Siguiendo un modelo de interpretación propuesto por Ricoeur(20-23), la OC en la atención en salud originaría situaciones de conflicto creciente, al contraponerse la aspiración entre los diferentes actores, representados por el profesional objetor (el "yo"); el paciente y el equipo (el "tú") y la misión y objetivos de la institución ("el otro sin rostro"). El conflicto se presentaría frente a la incompatibilidad entre este "deseo de ser" y la obligación, representada por la responsabilidad esperada de un profesional inmerso en una sociedad, resultando indispensable el resguardo del derecho por el logro de la vida buena de todos los involucrados, evitando que la toma de decisiones ocasione un daño irreparable, en el que la objeción se plantee como una excepción, considerando el respeto por el otro sobre la base de su propia libertad.

La unilateralidad o arbitrariedad de nuestras acciones o decisiones solo generarán y perpetuarán el conflicto. El equilibrio debe y puede lograrse mediante la sabiduría práctica, evaluando de manera racional y deliberando sobre las consecuencias que pudieran afectar la vida de los implicados, particularmente de los más vulnerables, en este caso, adolescentes consultantes en SSR.

La ética cívica $(24,25)$ proporcionaría las herramientas mediante la consideración de mínimos morales, traducidos en aquellos valores que determinan un mínimo ético irrenunciable, exigible moralmente a la totalidad de la población. Igualmente, debemos incluir las máximas morales, correspondientes a la aspiración al logro de la vida buena, de los diferentes actores o extrańos morales. La genuina OC estaría representada por este nivel de máxima moral, al ser invocada como una convicción por el objetor, como algo imposible de renunciar para el logro de esta aspiración. El nivel de mínimos se representaría por la necesidad de normar, de trabajar en conjunto, respetando y resguardando el derecho y libertad de todos los que de una u otra forma, directa o indirectamente, somos partícipes.

Al igual que en la sociedad, debemos transitar unidos hacia un entendimiento entre objetores y no objetores, sin exclusiones de aquellos que piensan distinto, sino de manera inclusiva, donde el diálogo, con apertura para generar puntos de encuentro, sea un esfuerzo que debamos promo$\operatorname{ver}(19)$.

\section{Agradecimientos}

A la profesora Nina Horwitz, por su constante apoyo y orientación, y al profesor Manuel Castillo, por su gentileza y generosidad. También a los profesionales entrevistados, por su tiempo y motivación. 
La objeción de conciencia de los profesionales en salud sexual y reproductiva de adolescentes - Adela Montero Vega

\section{Referencias}

1. Sieira S. La objeción de conciencia sanitaria. Madrid: Editorial Dykinson; 2000: 247.

2. Associació Catalana D’ Estudis Bioètics (ACEB). Objeción de conciencia en materia de salud. Disponible en http://www. aceb.org Acceso 15 de septiembre de 2014.

3. Araujo J. La objeción de conciencia al servicio militar. Madrid: Editorial Civitas;1993: 29.

4. Domínguez O. Objeción de conciencia, la muerte y el morir en enfermedades en etapa terminal. Acta Bioethica 2009; 15(1): 94-99.

5. Martínez K. Medicina y objeción de conciencia. Anales del Sistema Sanitario de Navarra 2007; 30(2): $215-223$.

6. Millaris Aparisi A, Guzmán López J. El Derecho a la Objeción de Conciencia en el supuesto del aborto. De la fundamentación filosófico-jurídica a su reconocimiento legal. Persona bioética 2005; 9 (26): 35-51.

7. Casas L, Dides C. Objeción de Conciencia y Salud Reproductiva en Chile: dos casos paradigmáticos. Acta Bioethica 2007; 13(2): 199-206.

8. Cook R, Arango M, Dickens B. Ethical and legal issues in reproductive health. Healthcare responsibilities and conscientious objection. International Journal of Gynecology and Obstetrics 2009; 104: 249-252.

9. Figo Committee Report. Ethical guidelines on conscientious objection. FIGO Committee for the Ethical Aspects of Human Reproduction and Women's Health. International Journal of Gynecology and Obstetrics 2006; 92: 333-334.

10. March JC, Prieto MA, Hernán M, Solas O. Técnicas cualitativas para la investigación en salud pública y gestión de servicios de salud: algo más que otro tipo de técnicas. Gac Sanit 1999; 13: 312-319.

11. De la Cuesta Benjumea C. Teoría y Método. La Teoría Fundamentada como herramienta de análisis. Cultura de los Cuidados 2006; 20: 136-140.

12. Dantas CC, Leite JL, Lima SB, Stipp MA. Teoría Fundamentada en los datos. Aspectos conceptuales y operacionales: metodología posible de ser aplicada en la investigación en Enfermería. Rev Latino - am Enfermagem 2009; 17(4).

13. Jones D, Manzelli H, Pecheny M. Grounded Theory: una aplicación de la Teoría Fundamentada a la salud. Cinta de Moebio 2004; 12.

14. Osses S, Sánchez I, Ibañez F. Investigación cualitativa en educación. Hacia la generación de teoría a través del proceso analítico. Estudios pedagógicos 2006; 32(1): 119-133.

15. Fernández MJ. Introducción a los Métodos Cualitativos de Investigación en Salud. Tema 2: Diseño de estudios y diseños muestrales en investigación cualitativa. Santiago de Chile: Material docente, Escuela de Salud Pública; 2010.

16. Mogollón A, Vásquez L. Introducción a los Métodos Cualitativos de Investigación en Salud. Tema 3: Técnicas cualitativas aplicadas en salud. Santiago de Chile: Material docente, Escuela de Salud Pública; 2010.

17. Amezcua M, Gálvez T. Los métodos de análisis en investigación cualitativa en salud: perspectiva crítica y reflexiones en voz alta. Rev. Esp. Salud Pública 2002; 76(5): 423-436.

18. Diccionario de la Real Academia Española. Disponible en http://www.rae.es/rae.html.

19. Montero A, González E. La objeción de conciencia en la práctica clínica. Acta Bioethica 2011; 17(1): 123-131.

20. Escríbar A. Aporte ricoeuriano a la bioética. Ciclo de Bioética Fundamental del Magíster de Bioética. Santiago de Chile: Universidad de Chile, Facultad de Filosofía y Humanidades, Apuntes de clases presenciales, 20 semestre 2009.

21. Escríbar A. Raíces de la Bioética en la tradición ético-filosófica occidental y actuales desafíos. Anales de La Universidad de Chile 1998; 6a serie; 8: 1-20.

22. Escríbar A. La ética aplicada, sus condiciones de posibilidad y exigencias a las que responde. Revista de Filosofía 2004; 60: 19-28.

23. Ricoeur P. Sí mismo como Otro. Séptimo estudio: El Sí y la intencionalidad ética. Traducción Agustín Neira. Madrid: Siglo XXI; 1996.

24. Cortina A. Ética mínima como Ethica cordis. En Ética de la razón cordial. Educar en la ciudadanía en el siglo XXI. Madrid: Ediciones Nobel S. A; 9-32.

25. Chávez P. Ética cívica: tiempo de sumar, no de restar. Rev. Mensaje 2007: 56(557): 38-41.

Recibido: 25 de mayo de 2013

Aceptado: 10 de septiembre de 2013 